\title{
27
}

\section{Measuring the Value and Performance of Your IT Department}

\author{
Terry Lynch
}

Western Sydney Area Health Service, Sydney, Australia 2151

\begin{abstract}
QUALITY is a cost usually overlooked in software estimation and software valuation. This paper outlines the software quality metrics initiatives developed and being deployed at Western Sydney Area Health Service (WSAHS), Sydney, Australia to address these issues.

WSAHS is a unit of the NSW Health Department employing 8,500 staff in 7 hospitals, 6 community health centres and a dental school. WSAHS has a patient catchment of 1.4 million and is one of the largest VAX and Novelle sites in Australia. WSAHS IS Department has a staff of 56 or whom 20 are software developers working on VAX and PC Workstation applications.

WSAHS needed indicators to demonstrate compliance to quality requirements when delivering systems and to enable corporate planners to monitor trends in software costs. We need also, indicators to use during the software development cycle to determine the cost of quality when negotiating software development and maintenance project funding.
\end{abstract}

\section{OBJECTIVES}

To begin with the objectives for software quality indicators were defined as follows:

*To be able to conduct objective evaluations of the quality of software products.

*To provide a measurement to enable the negotiation of the level of quality in a system prior to development beginning.

*To be able to quantify quality so that it can be built into development estimates

*To be able to quantify the quality of production systems to support strategic decisions for resource utilisation and the system replacement planning.

*To be able to predict the production quality of a product whilst still in development and dynamically take steps to ensure that the quality of a product satisfies and predetermined standard.

*To gauge the effectiveness of quality initiatives.

Only the first three of these criteria have been addressed at the time.

\section{METRIC CHARACTERISTICS}

Having established quality objectives, we established the guidelines for the metrics as being:

a. Objective. The metric must be derivable by a repeatable, quantifiable and auditable process. It must not be intuitive or subjective.

b. Linked to a business need. That is the data has to be gathered for a purpose. That is there must be a clearly defined reason for gathering the data and management must be prepared to act on the indications from the data.

c. Useful. That is to say the data from the metric must be timely and sensitive to of any change in the behaviour or practices the metric is meant to monitor.

d. Economical and easy to gather. If the gathering of the metric data is costly and difficult it will 
eventually become too much trouble to gather. Especially if there is no perceived benefit to those gathering the data (ie the project teams) and the sponsor or the metrics program. To this end, we designed and constructed three metrics tools in conjunction with the University of Western Sydney. Those tools being an Function Point Tool, a two method estimation tool and a Quality Tracking System. All these tools link to a Project History Data Base and MSProject tracking tool.

e. Have a Counter Metric. For every metric there has to be a counter metric so that the metrics do not divert the focus of an organisation away from their primary purpose to a secondary purpose - satisfying the metrics.

g. Normalisation. Since the size and complexity of software is so variant, it was recognised that a method of normalising of results be determined. Without it any comparison between projects will be difficult because an allowance cannot be made for size and of products developed and applications developed on different platforms. The normalisation factors choosen were:

- size (function points)

- complexity

- frequency of use and

- the number of users of a system.

\section{What to measure?}

We identified two distinct groups of metrics we needed to consider to satisfy our objectives:

- Metrics which help us manage the development process and

- Metrics which indicate fit for purpose of software products.

Within those categories we have two sub-categories - Effectiveness and Efficiency.

The efficiency of a software products being how well a product utilises resources (both hardware, consumables and human effort). The effectiveness of a product being how well the product meets the current business need.

For the development process - the software assembly line the objective was to to determine if our tools and techniques are effective and whether we are using them as efficiently as we might.

At this time we have focused on the effectiveness of our products and processes.

\subsection{Product Metrics}

The measurement criteria of product efficiency and effectiveness chosen was the USAF Rome Laboratories Quality Criteria or RADC Criteria. In this process eleven criteria are identified.

- Conformity to requirements and the business need

- Ease of use

- Reliability

- Flexibility or ease of change

- Maintainability

- Reusability of modules of logic in the system

- Efficiency in execution and utilisation of hardware

- Integrity and security of a system and its data

- Interoperability or the ability of the software to interact with other software in use in the organisation

- Portability of the software to other platforms.

- The ease of testing the software.

Non Functional requirements are defined at the requirements gathering phase of all projects and Business Realisation Reviews (BRR) are conducted 12 months after implementation examine the same 
criteria. The BRR criteria are each rated from 1 being poor to 5 being excellent the mean score being a Quality Index. All systems are required to meet a QI rating of 3.75 or better. Since introducing this scoring system, four BRRs have been conducted and their results are shown below.

However, the scoring did highlight consistently low ratings for user documentation which is now being addressed.

\subsubsection{Other metrics}

In addition the following measures have been identified and raw data is now being collected. However, these metrics can not be collated until October when the Function Point Tool comes online.

Ease of Use. How user friendly is the software? The measure used was the number of requests for help received at the help desk. High volumes of request would suggest that the software is difficult to use.

Help desk calls

Function points $x$ number of users $x$ hours of operation

Interoperability. How easy is it for end users to switch between applications? This is be a major concern of clinicians and health workers dealing with the public.

Average steps to exit an application.

The following product efficiency attributes were taken from the IFPUG Function Point Asset Management manual and will :

Reliability. What is the average time to failure of a product? How expensive is it to support a software product compared to other software.

Reliability = cost of repair/ size( $\mathrm{x}$ complexity)

Completeness. How often are requests for change received from the clients.

Completeness $=$ Requests for change/ size

Maintainability. What is the application support rate and what is the trend of the costs for a software product.

Maintainability $=$ Maintenance costs $/$ size

\subsection{Process Metrics}

As mentioned earlier, the metrics gathered need to fit a purpose. The objectives of the quality metrics in the development environment were to:

- Identify good and bad development practices and to introduce practices to promote the good initiatives and impeded counter productive practices.

- Be able to negotiate quality levels to reflect the business need of the organisation.

The development process metrics chosen are :

Testing Proficiency $=$ Defects discovered

$$
\text { Size } x \text { Complexity }
$$

Efficiency of the process $=$ Defects introduced from previous phases

Total review issues for a phase $\mathrm{x}$ size

A defect being an error requiring re-work. For example, a design error not detected until programming.

Again until the FPA tool is ready, these formulations can not be applied however, comparisons of Defects subjectively have highlighted some key issues to address in Requirements gathering. These problems related to our use of JAD sessions and ER-Modelling that had to be addressed immediately.

\section{IMPLEMENTATION TACTIC}

The first activity was to review metrics programs in a major financial institution and a major Australian airline to establish what were the critical success factors for their metrics programs. This 
identified 9 critical considerations:

- Sustained management sponsorship. If there is not a strong commitment to the measurement program from the very top then commitment from the metric gatherers will wane.

- The metrics had to be linked to the business needs of the organisation. If there is no purpose for gathering the metrics and no action is taken on trends that the indicators are showing then commitment and enthusiasm and eventually funding will dissipate.

- The metrics had to be relevant to all involved and understood by the players.

- Metrics must not be perceived to be unfair and therefore resisted.

- Care had to be taken not to motivate the wrong behaviour

- Metrics collection must be inexpensive and not cumbersome to collect

- Must act on the indications the metrics are showing.

- Do not try to introduce too many metrics at once. Try to keep the metrics simple and uncomplicated so that they are easily understood.

- Where possible use graphics to represent data and automate the data collection.

\section{EARLY INDICATIONS}

The development of the tool set in conjunction with the University of Western Sydney has proven to be a critical success factor. For example early attempts to introduce manual Function Point counting proved unsuccessful due to the time consumed and lack of consistency. Likewise the completion of review statistics always required close supervision. When projects began to slip the review details were often not completed. By providing a high level view of review progress and having the review events being designated by a system, reviews could not be missed and had to be completed.

Heavy post implementation support indicated inadequacies in requirements gathering and testing procedures. The defect tracking confirmed this issue enabling corrective action to be initiated to improve staff Entity Relationship Modelling and conducting JAD sessions. 